\title{
CARACTERIZAÇÃo FÍSICA, gUÍmiCA, MicrobiológICA E SENSORIAL DE GELÉIAS LIGHT DE ABACAXI ${ }^{1}$
}

\author{
Graziele Guimaraes GRANADA²; Rui Carlos ZAMBIAZI ${ }^{3}$; \\ Carla Rosane Barboza MENDONÇA ${ }^{4}$; Edilson SILVA ${ }^{5}$
}

\section{RESUMO}

O trabalho objetivou elaborar geléias com reduzido teor calórico, utilizando como agentes de corpo diferentes combinações dos hidrocolóides xantana, carragena e locusta. Como comparativo utilizou-se uma formulação padrão de geléia de abacaxi (controle), elaborada com sacarose e xarope de glicose na proporção de 4:1 (p/p), e com teor final de sólidos solúveis de $65^{\circ} B r i x$. As quatro formulações light foram preparadas pela substituição de $50 \%$ da quantidade de açúcar da formulação controle, sendo que para equiparar a doçura, adicionou-se o edulcorante sucralose. Foram avaliadas as características químicas, físicas, microbiológicas e sensoriais, além do valor calórico total das geléias. Os resultados mostraram que as geléias apresentaram características químicas e físicas semelhantes às descritas na literatura e enquadraram-se na categoria de produtos light, segundo a legislação vigente. Quanto às características microbiológicas, todas as formulações enquadraram-se nos padrões microbiológicos estabelecidos pela legislação brasileira. Em termos sensoriais a formulação com a combinação das gomas carragena:xantana:locusta (1:1:1, p/p/p) foi a que apresentou melhores características de geléia.

Palavras-chave: geléia de baixo valor calórico, xantana, locusta, carragena.

\section{ABSTRACT}

PHYSICAL, CHEMICAL, MICROBIOLOGICAL AND SENSORY CHARACTERIZATION OF LIGHT JELLIES OF PINEAPPLE. The objective of the work was to elaborate jellies with reduced caloric content, by using as body replacers a combination of the hydrocolloids xanthan, carrageenan and locust. The pineapple jelly was used as control formulation, and it was made with sucrose and glucose in the proportion of $4: 1 \mathrm{p} / \mathrm{p}$, and soluble solid content of $65^{\circ} \mathrm{Brix}$. The four light formulations were prepared by substitution of $50 \%$ of the amount of sugar of the control formulation and, to compensate the sweetness, the low-calorie sweetener sucralose was used. The chemical, physical, microbiological, sensory and the total caloric content of the jellies were evaluated. The results showed that the jellies presented chemical and physical characteristics similar to the others presented in the literature, and they were classified in the category of light products, according to the running legislation. All the formulations met the standard microbiological specifications of the Brazilian legislation. The formulation with the combination of the gums carrageenan:xanthan:locust ( $1: 1: 1, \mathrm{p} / \mathrm{p} / \mathrm{p}) \mathrm{was}$ the one that presented better characteristics of jelly.

Keywords: light pineapple jelly, xanthan, carrageenan, locust.

\section{1 - INTRODUÇÃO}

Problemas de saúde como obesidade, diabetes, hipertensão ou mesmo preocupações com a estética corporal têm estimulado a pesquisa e o desenvolvimento de produtos de baixo valor calórico [26]; para tanto, uma série de edulcorantes e espessantes estão sendo utilizados no Brasil [27].

A formulação de um produto de teor calórico reduzido deve iniciar pelo exame detalhado do produto tradicional [26]. Produtos com reduzido teor de sólidos solúveis, como geléias light, são suscetíveis à sinérese, textura frágil [43], falta de limpidez, perda de coloração e sabor; neste sentido, faz-se necessário aprofundar as pesquisas com a utilização de gomas apropriadas, visando melhorar as características reológicas e amenizar os problemas inerentes à redução de sólidos nestes produtos [41, 14].

As gomas são compostos poliméricos que, quando dissolvidos ou dispersos em água, formam soluções ou dispersões viscosas. Pertencem ao grupo dos hidrocolóides

\footnotetext{
${ }^{1}$ Recebido para publicação em 07/01/2002. Aceito para publicação em 31/08/2005 (000793).

${ }^{2}$ Mestre em Ciência, Departamento de Educação Física e Saúde, UNISC.

${ }^{3}$ Professor, Doutor em Food and Nutritional Science, Departamento de Ciência dos Alimentos/UFPEL.

${ }^{4}$ Mestranda em Ciência e Tecnologia Agroindustrial/Departamento de Química Orgânica/UFPEL.

${ }^{5}$ Graduando em Química/IQG/UFPEL.
}

ou seus derivados, os quais possuem ampla aplicação como agentes espessantes e estabilizantes. Podem ser obtidos a partir de extratos de algas marinhas (alginatos, ágar, carragenas), extratos de sementes (locusta, guar), exsudatos vegetais (arábica), microrganismos (xantana, gelana) e a partir de celulose e pectina [33].

Estes produtos possuem a capacidade de reter grande quantidade de água, proporcionam baixos índices de calorias e acrescentam características de textura e sensação tátil bucal aos substitutos de gordura. Além disto, auxiliam na estabilização de emulsões e suspensões de partículas, no controle da cristalização, inibição de sinérese, encapsulamento e formação de filmes comestíveis [19, 26].

Os hidrocolóides já fazem parte da formulação de vários produtos manufaturados, tendo seu uso consagrado pela indústria alimentícia, onde são utilizados com diferentes propósitos; no entanto, sua aplicação em alimentos light e diet ainda está sendo estudada.

A goma xantana é amplamente utilizada na indústria de alimentos devido às suas propriedades de emulsificação, suspensão, estabilização e floculação e mantém estas características em uma ampla faixa de $\mathrm{pH}$ e temperatura [1]. Associada a outras gomas, como a locusta (jataí), proporciona textura lisa e cremosa a alimentos líquidos, com qualidade superior a outros espessantes; também apresenta, como vantagem, um baixo valor calórico 
(0,5 kcal/g), porque somente $15 \%$ desta goma é digerido pelos processos metabólicos no organismo humano [9].

A goma locusta é um polissacarídeo neutro que isoladamente não forma gel, mas pode fazê-lo junto à xantana e à k-carragena. Atua como espessante, estabilizante de emulsões e inibidor de sinérese e também apresenta estabilidade na faixa de $\mathrm{pH}$ de 3,5 a 11,0 [10, 12].

Carragena é o nome genérico aplicado a hidrocolóides extraídos de algas vermelhas [4], constituídos de unidades de galactose e anidrogalactose, constituindo-se em um polímero sulfatado. Em função do conteúdo e distribuição dos grupos de ésteres sulfatados, as carragenas podem ser classificadas em iota, kappa e lambda [31; 32]. Esta goma atua como emulsificante, geleificante, estabilizante e possui a capacidade de manter partículas em suspensão. A máxima estabilidade é atingida em pH 9,0, não devendo ser processada a quente em pH inferior a 3,5; no entanto, em pH superior a 6,0 , resiste às condições normais de esterilização. O uso de carragenas é indicado para formulação de medicações e em diversos produtos de baixo teor calórico, como em queijos, produtos cárneos, pudins, chocolates e geléias [9].

A utilização de hidrocolóides isolados ou associados como agentes geleificantes permite a implementação das propriedades reológicas, beneficiando as características dos produtos alimentícios formulados para fins especiais, bem como de geléias light [41]. A gelatinização ocorre quando uma solução aquosa do polímero sofre resfriamento, presumivelmente pela formação de estrutura dupla-hélice para produzir rede polimérica tridimensional [16].

O presente trabalho teve como objetivo elaborar geléias de abacaxi light, substituindo parcialmente o açúcar por edulcorantes, repondo o corpo pela adição das gomas xantana, carragena e locusta; além de caracterizar física, química, sensorial e microbiologicamente estes produtos, comparando-os a uma geléia convencional.

\section{2 - MATERIAL E MÉTODOS}

\section{1 - Materiais}

Para elaboração das geléias foram utilizados abacaxi da cultivar cayenne (oriundo da região do Triângulo Mineiro, safra 2000, cedido pela Indústria de Conservas Icalda/ RS), sacarose e xarope de glicose (adquiridos no comércio local), pectina (com $150^{\circ} \mathrm{SAG}$ ), ácido cítrico, edulcorante sucralose (sob a forma líquida na concentração de 3,1\%, marca comercial Splenda), e as gomas locusta (marca comercial Viscogum FA), xantana (marca comercial Satiaxane CX 91) e carragena (marca comercial Satiagel OF 10), todas doadas pela SKW BIOSISTEM.

\section{2 - Métodos}

\subsection{1 - Processo de elaboração das geléias}

A elaboração das geléias de abacaxi foi realizada na linha de processamento industrial (Figura 1) da Fábrica de Conservas Icalda, localizada em Pelotas-RS, em outubro de 2000.

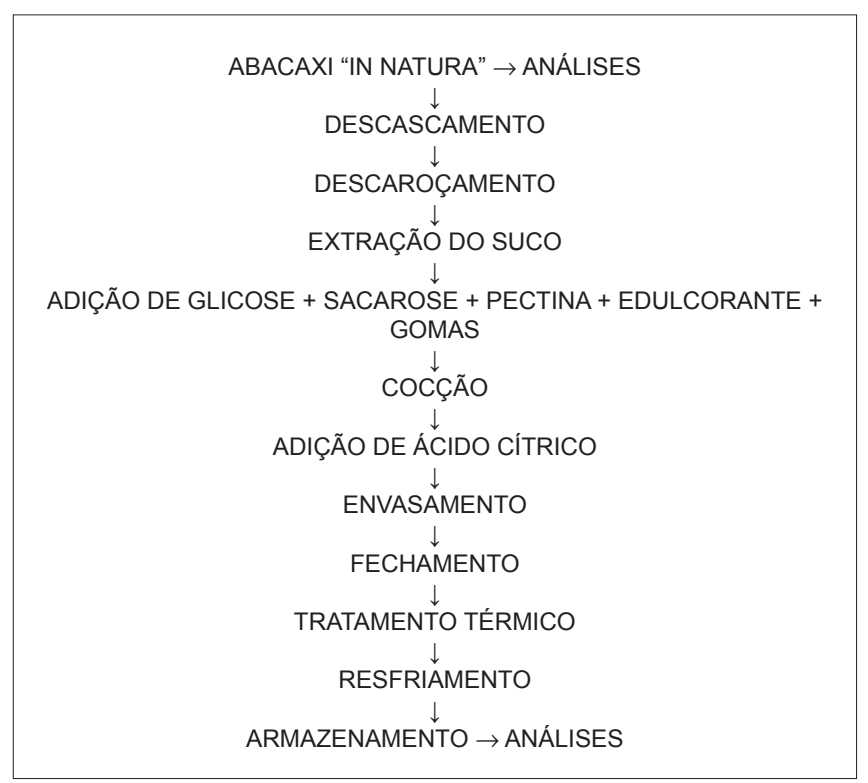

FIGURA 1 - Fluxograma do processamento de geléias com reduzido teor de sólidos solúveis

O abacaxi inicialmente passou pelas operações mecânicas de descascamento e retirada da parte central, seguido do corte manual da polpa. A polpa foi fervida com água à pressão atmosférica, na proporção de $2: 1, \mathrm{p} / \mathrm{p}$, até obtenção do suco a $10^{\circ} \mathrm{Brix}$, e posteriormente filtrada com auxílio de peneiras de 50 mesh.

Ao suco de abacaxi, foram adicionadas sacarose e glicose (4:1 p/p em relação à massa total de xarope) e pectina (quantidade calculada com base no ${ }^{\circ} \mathrm{SAG}$ e peso de sacarose). A formulação da geléia utilizada como controle (F1) foi elaborada segundo as indicações do SENAI [34], considerando as proporções para uma geléia do tipo extra.

Para as formulações light, substituiu-se 50\% do açúcar pelo edulcorante sucralose, calculado considerando-se a doçura equivalente, conforme mostra a Tabela 1 , porém respeitando o limite máximo de $45 \mathrm{mg}$ de edulcorante por 100 g de produto final, além de adicionar-se as gomas ( $2 \%$ sobre o peso da sacarose).

TABELA 1 - Relação entre sacarose e sucralose

\begin{tabular}{lcccc}
\hline Substância & $\begin{array}{c}\text { Doçura } \\
\text { relativa }\end{array}$ & $\begin{array}{c}\text { Quantidade } \\
\text { (g/kg de } \\
\text { sacarose) }\end{array}$ & $\begin{array}{c}\text { Limite máximo } \\
\text { em alimentos }\end{array}$ & Calorias/g \\
\hline Sacarose & 1 & - & - & 4 \\
Sucralose & 600 & 1,67 & 45 & 0 \\
\hline
\end{tabular}

Fonte: Cândido \& Campos [8]

${ }^{* 1} \mathrm{mg}$ de edulcorante por $100 \mathrm{~g}$ de produto alimentício

As formulações light foram preparadas pela adição dos hidrocolóides, nas seguintes combinações: xantana e locusta ( $1: 1, \mathrm{p} / \mathrm{p}-\mathrm{F} 1)$, carragena e xantana ( $1: 1, \mathrm{p} / \mathrm{p}-\mathrm{F} 2)$, 
carragena e locusta ( $1: 1, \mathrm{p} / \mathrm{p}$ - F3) e carragena, xantana e locusta $(1: 1: 1, \mathrm{p} / \mathrm{p} / \mathrm{p}-\mathrm{F} 4)$.

A cocção se deu em tacho aberto (modelo TC-150), usando misturador de pá elétrica. Para todas as formulações adicionou-se o ácido cítrico (0,5\% sobre o peso do açúcar) momentos antes de atingir o ${ }^{\circ}$ Brix final.

A formulação controle foi preparada com a concentração final de sólidos solúveis de $65^{\circ} \mathrm{Brix}$ e as formulações light, com concentrações de sólidos solúveis entre $45-47^{\circ} \mathrm{Brix}$.

Os produtos foram acondicionados em embalagens de vidro, previamente esterilizadas a $100^{\circ} \mathrm{C} / 15 \mathrm{~min}$ com capacidade para $250 \mathrm{~g}$ e fechadas com tampa de metal. O tratamento térmico foi efetuado em banho-maria a $100^{\circ} \mathrm{C}$ por 15 minutos, em seguida os produtos foram resfriados por adição de água fria por 15 minutos e posteriormente armazenados à temperatura ambiente.

\subsection{2 - Análise física e química}

As geléias foram analisadas quanto ao teor de proteínas, gordura, $\mathrm{pH}$, acidez, sólidos solúveis, açúcares totais, açúcares redutores e açúcares não-redutores. As análises foram realizadas em triplicata, de acordo com os procedimentos indicados pelas Normas Analíticas do Instituto Adolfo Lutz [20].

Com base nas determinações químicas, realizou-se também a determinação do valor calórico total, seguindo a metodologia descrita por Franco [13].

\subsection{3 - Análise microbiológica}

Determinou-se o Número Mais Provável de coliformes totais e fecais (NMP.g ${ }^{-1}$ ) e a contagem de bolores e leveduras $\left(\mathrm{UFC} \mathrm{g}^{-1}\right)$ para todas as geléias. Estas análises foram realizadas seguindo os procedimentos descritos pelo Compendium for the Microbiological Examination of Foods [42].

\subsection{4 - Análise sensorial}

As geléias foram avaliadas quanto aos atributos de aparência, cor, brilho, consistência do gel, odor, sabor característico, sabor estranho, doçura e acidez, por uma equipe de 9 julgadores treinados [2].

Para a seleção e o treinamento da equipe, foram realizados os testes dos gostos primários (ácido, doce, metálico); limiar absoluto para gosto ácido; ordenação de amostras por intensidade de cor; escala de categorias; pareado-grau de diferença e avaliação de atributos, de acordo com a Associação Brasileira de Normas Técnicas [3] e International Organization for Standardization [24; 25]. Cerca de 30 sessões foram realizadas no período de três meses, em cabines individualizadas, conforme indicação da International Organization for Standardization [23].

O julgamento das amostras foi realizado pelo teste de avaliação de atributos, a partir de uma escala de intervalo não estruturada de $9 \mathrm{~cm}$, conforme as indicações da ISO [22]. Os 9 atributos avaliados foram Aparência, Cor e Odor, sendo que o extremo zero representou "nada característico a geléia" e nove representou "característico a geléia"; Brilho, Sabor Estranho e Sabor Característico, tendo como extremo zero o termo "não-perceptível" e o extremo nove como "forte"; Consistência do Gel, sendo que o valor zero caracterizou "gel mole" e o valor nove caracterizou "gel firme"; Doçura e Acidez, com o extremo zero para "nãoperceptível" e o outro extremo como "muito doce e muito ácido", respectivamente.

As amostras foram apresentadas aos julgadores em potes de porcelana branca, codificados com três algarismos aleatórios. Cada amostra foi constituída por cerca de 20 g de geléia, à temperatura ambiente, de acordo com as recomendações da International Organization for Standardization [21].

\section{3 - Delineamento estatístico}

Os resultados foram avaliados pela análise de variância (ANOVA), sendo que os resultados que mostraram diferenças significativas de médias foram complementados com o teste de Tukey, todos ao nível de $5 \%$ de probabilidade, pelo programa Statistica 5.1 [35].

\section{3 - RESULTADOS E DISCUSSÃO}

A Tabela 2 mostra os resultados obtidos nas determinações físicas e químicas efetuadas nas geléias de abacaxi.

TABELA 2 - Determinações físicas e químicas das geléias de abacaxi

\begin{tabular}{|c|c|c|c|c|c|}
\hline & \multicolumn{5}{|c|}{ Geléias } \\
\hline & F1 & F2 & F3 & F4 & F5 \\
\hline Proteína (g \%) & $0,28 \mathrm{a}$ & $0,26 a b$ & $0,21 \mathrm{~b}$ & $0,22 a b$ & $0,26 a b$ \\
\hline Gordura (g \%) & 0,13 ac & $0,11 \mathrm{ad}$ & $0,16 \mathrm{c}$ & 0,07 bde & 0,09 ae \\
\hline $\begin{array}{l}\text { Açúcares totais } \\
\text { (\% glicose) }\end{array}$ & $41,73 \mathrm{a}$ & $40,57 \mathrm{~b}$ & $40,84 \mathrm{~b}$ & $39,74 \mathrm{c}$ & $63,04 \mathrm{~d}$ \\
\hline $\begin{array}{l}\text { Açúcares redutores } \\
\text { (\% glicose) }\end{array}$ & $14,50 \mathrm{a}$ & $14,64 \mathrm{a}$ & $19,33 \mathrm{~b}$ & $14,90 \mathrm{a}$ & $20,02 \mathrm{~b}$ \\
\hline $\begin{array}{l}\text { Açúcares não redutores } \\
\text { (\% sacarose) }\end{array}$ & $25,87 a$ & $\begin{array}{c}24,64 \\
\text { ac }\end{array}$ & $20,44 \mathrm{~b}$ & $24,02 \mathrm{c}$ & $40,86 \mathrm{~d}$ \\
\hline Sólidos solúveis ('Brix) & $47,00 \mathrm{a}$ & $45,20 \mathrm{~b}$ & $46,00 \mathrm{c}$ & $46,20 \mathrm{c}$ & $65,10 \mathrm{~d}$ \\
\hline $\mathrm{pH}$ & $3,53 \mathrm{ac}$ & $3,56 a b$ & $3,58 \mathrm{~b}$ & $3,56 a b$ & $3,50 \mathrm{c}$ \\
\hline $\begin{array}{l}\text { Acidez } \\
\text { (\% de ác. cítrico) }\end{array}$ & $0,85 \mathrm{a}$ & $0,87 \mathrm{ab}$ & $0,90 a b$ & $0,95 b$ & $0,75 \mathrm{c}$ \\
\hline Calorias (kcal/100 g) & 169 a & $164 \mathrm{~b}$ & $166 \mathrm{~b}$ & $161 \mathrm{c}$ & $254 d$ \\
\hline
\end{tabular}

F1 (1:1, p/p, xantana/ locusta)

F2 (1:1, p/p, carragena/ xantana)

F3 ( $1: 1, \mathrm{p} / \mathrm{p}$, carragena/ locusta)

F4 (1:1:1, p/p/p, carragena/ xantana/ locusta)

F5 (formulação controle)

Letras diferentes na mesma linha evidenciam diferenças significativas ao nível de $5 \%$ de probabilidade pelo teste de Tukey $(\mathrm{p}<0,05)$

Pelos resultados observa-se que a formulação controle (F5) apresentou a menor acidez, diferindo significativamente das demais. Aparentemente não se observou influência da associação das gomas sobre a acidez das geléias. Os valores de acidez das formulações light $(0,85-0,95)$ foram bem simi- 
lares aos encontrados em geléias de manga processada sem adição de açúcar, que foi de 0,91\% em ácido cítrico [39].

Embora apresentando a menor acidez, a amostra controle (F5) também mostrou o menor valor de pH, mas não diferindo significativamente da formulação F1. Os valores de $\mathrm{pH}$ encontrados para todas as formulações foram mais elevados do que os valores encontrados por CAMPOS \& CÂNDIDO [7] em geléias de abacaxi com reduzido teor de sólidos solúveis, as quais foram formuladas com o uso de diversos edulcorantes e em diferentes composições, sendo que os autores obtiveram valores de $\mathrm{pH}$ entre 3,10 a 3,25 .

Tanto para os valores de proteína quanto de gordura não ocorreram variações marcantes entre as diferentes geléias, o que era esperado, pois apenas alterações na quantidade de açúcar e de gomas nas formulações não poderiam interferir no conteúdo destes componentes. Os valores encontrados para proteína tiveram grande similaridade aos obtidos em geléias convencionais de abacaxi e de marca comercial $(0,26 \mathrm{~g} \%)$, porém bem abaixo das dietéticas do mesmo fabricante $(0,47 \mathrm{~g} \%)$. Por outro lado, o conteúdo de gordura apresenta-se bem inferior ao encontrado em geléias dietéticas $(0,50 \mathrm{~g} \%)$ [15].

Comparando os resultados das 4 formulações light deste estudo com os valores encontrados em estudo específico de geléias light de morango, pêssego, ameixas e abacaxi [37], em que os pesquisadores obtiveram uma redução entre 20,8 a 33,9\% no teor de açúcares totais, observou-se uma redução semelhante (F1 - 33,80\%) e até um pouco maior (F2 - 35,64\%; F3 - 35,22\%; F4 - 36,96\%), em relação aos açúcares totais. Valores aproximados na redução do conteúdo total de açúcares $(32,82 \%)$ foram obtidos em geléias de maçã com sucralose, nas quais também foi testada uma substituição de $50 \%$ da sacarose [28].

Pela Figura 2, observa-se que os valores calóricos das geléias light de abacaxi ficaram entre 161 a 169 kcal/100 g; sendo que o valor calórico da formulação convencional foi de $254 \mathrm{kcal} / 100 \mathrm{~g}$.

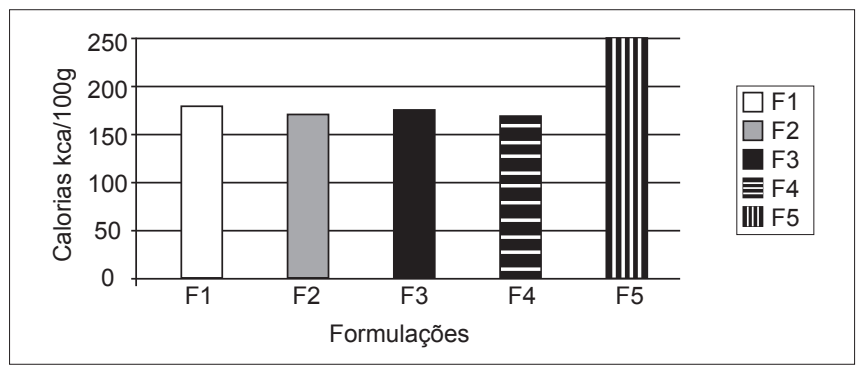

Figura 2

A redução de calorias das formulações light em relação à controle foi de 33,38; 35,3; 34,78 e 36,81\%, para as formulações F1, F2, F3 e F4, respectivamente. Esta redução atendeu à legislação brasileira [5], que menciona a redução mínima de $25 \%$ no conteúdo de açúcares.

A Tabela 3 apresenta os resultados encontrados nas análises microbiológicas das geléias de abacaxi.
TABELA 3 - Características microbiológicas das geléias de abacaxi

\begin{tabular}{lccccc}
\hline & \multicolumn{5}{c}{ Geléias } \\
\cline { 2 - 6 } & F1 & F2 & F3 & F4 & F5 \\
\hline $\begin{array}{l}\text { Coliformes totais } \\
\left.(\text { NMP.g })^{-1}\right)\end{array}$ & $<3$ & $<3$ & $<3$ & $<3$ & $<3$ \\
$\begin{array}{l}\text { Coliformes fecais } \\
\left(\text { NMP.g } \text { - }^{-1}\right.\end{array}$ & $<3$ & $<3$ & $<3$ & $<3$ & $<3$ \\
$\begin{array}{l}\text { Bolores e leveduras } \\
\left(\text { UFC. } g^{-1}\right)\end{array}$ & $<10^{4}$ & $<10^{4}$ & $<10^{4}$ & $<10^{4}$ & $<10^{4}$ \\
\hline
\end{tabular}

F1 (1:1, p/p, xantana/ locusta)

F2 (1:1, p/p, carragena/ xantana)

F3 (1:1, p/p, carragena/ locusta)

F4 (1:1:1, p/p/p, carragena/ xantana

F5 (formulação controle)

Pela Tabela 3 pode-se constatar que nenhuma das formulações apresentou contaminação por coliformes ou por bolores e leveduras; dessa forma, todas as formulações enquadraram-se nos padrões estabelecidos pela legislação vigente [6], a qual estabelece uma tolerância de $10^{4}$ UFC.g ${ }^{-1}$ para bolores e leveduras. Embora a RDC n ${ }^{\circ} 12$ não mencione metas a serem atingidas na pesquisa dos coliformes, os mesmos podem apontar deficiências no tratamento térmico, já que não são formadores de esporos [18].

NACHTIGALL et al. [30] e MENDONÇA et al. [29] também obtiveram resultados microbiológicos dentro dos padrões vigentes, nos quais constataram incidência e crescimento de microrganismos do grupo coliforme e de mofos e leveduras sempre inferiores a 3 NMP.g ${ }^{-1}$ e 30 UFC.g $^{-1}$, respectivamente.

Devido à baixa resistência térmica, os mofos e leveduras raramente estão associados a processos de deterioração de produtos que sofreram tratamento térmico; porém, deve ser lembrada a existência de algumas espécies termorresistentes de fungos deterioradores [39].

A Tabela 4 apresenta os resultados obtidos na avaliação sensorial das formulações de geléias light e da geléia convencional.

TABELA 4 - Características sensoriais das geléias de abacaxi

\begin{tabular}{lccccc}
\hline & \multicolumn{5}{c}{ Geléias } \\
\cline { 2 - 6 } & F1 & F2 & F3 & F4 & F5 \\
\hline Aparência & $1,98 \mathrm{a}$ & $3,80 \mathrm{a}$ & $4,06 \mathrm{a}$ & $6,78 \mathrm{~b}$ & $7,80 \mathrm{~b}$ \\
Cor & $3,80 \mathrm{a}$ & $4,17 \mathrm{ab}$ & $5,18 \mathrm{ac}$ & $6,57 \mathrm{bc}$ & $7,46 \mathrm{c}$ \\
Brilho & $2,59 \mathrm{a}$ & $6,28 \mathrm{bc}$ & $7,18 \mathrm{bc}$ & $5,92 \mathrm{~b}$ & $7,99 \mathrm{c}$ \\
Odor & $4,92 \mathrm{a}$ & $5,70 \mathrm{a}$ & $6,60 \mathrm{a}$ & $6,61 \mathrm{a}$ & $6,97 \mathrm{a}$ \\
Consistência do gel & $8,03 \mathrm{a}$ & $1,32 \mathrm{~b}$ & $2,43 \mathrm{~b}$ & $5,82 \mathrm{c}$ & $5,09 \mathrm{c}$ \\
Sabor característico & $2,64 \mathrm{a}$ & $4,82 \mathrm{ab}$ & $5,23 \mathrm{~b}$ & $5,61 \mathrm{~b}$ & $6,33 \mathrm{~b}$ \\
Sabor estranho & $1,49 \mathrm{a}$ & $1,61 \mathrm{a}$ & $1,10 \mathrm{a}$ & $1,43 \mathrm{a}$ & $0,81 \mathrm{a}$ \\
Doçura & $4,53 \mathrm{a}$ & $6,12 \mathrm{a}$ & $6,21 \mathrm{a}$ & $6,92 \mathrm{a}$ & $5,67 \mathrm{a}$ \\
Acidez & $2,42 \mathrm{a}$ & $5,64 \mathrm{~b}$ & $5,00 \mathrm{~b}$ & $5,01 \mathrm{~b}$ & $4,26 \mathrm{ab}$ \\
\hline
\end{tabular}

F1 (1:1, p/p, xantana/ locusta)

F2 (1:1, p/p, carragena/ xantana)

F3 (1:1, p/p, carragena/ locusta)

F4 ( $1: 1: 1, \mathrm{p} / \mathrm{p} / \mathrm{p}$, carragena/ xantan

F5 (formulação controle)

Letras diferentes na mesma linha evidenciam diferenças significativas ao nível de $5 \%$ de probabilidade pelo teste de Tukey $(\mathrm{p}<0,05)$

Pela Tabela 4 pode-se observar que para os atributos odor, sabor estranho e doçura, os julgadores não detectaram diferenças significativas entre as geléias, evidenciando grande similaridade entre as diferentes formulações quanto 
a estes atributos. Em estudo anterior, no qual empregouse o mesmo edulcorante (sucralose) em geléias de maçã com diferentes concentrações de açúcares e goma carboximetilcelulose, verificou-se que a doçura também não mostrou diferença significativa entre as formulações light e a controle [17].

Mesmo com evidente diferença na composição química entre a formulação controle e as formulações light, tanto nos sólidos solúveis como no conteúdo de açúcares (totais, redutores e não-redutores), os resultados da análise sensorial mostraram que os julgadores não perceberam diferenças de sabor, como o estranho, e doçura, comprovando os resultados obtidos por MENDONÇA [28] na substituição parcial da sacarose por sucralose, indicando a excelente performance deste edulcorante.

Em termos de sabor característico, as formulações F2, F3 e F4 não diferiram significativamente da formulação controle; situando-se na escala entre regular e forte. Já no atributo acidez, as geléias F2, F3 e F4 situaram-se entre regular e muito ácidas na escala. A formulação $\mathrm{F} 1$ foi a que apresentou maiores diferenças significativas entre as demais, indicando ser uma geléia pouco ácida e de menor sabor característico. Estes resultados estiveram relacionados à acidez titulável das geléias light. No entanto, o mesmo não ocorreu com a formulação controle, já que esta foi a de menor acidez em ácido cítrico e isto não se refletiu no painel sensorial.

Pelas notas atribuídas, as geléias F2, F3, F4 e Formulação Controle caracterizaram-se em relação ao brilho entre regular e forte, e novamente a formulação F1 diferiu significativamente de todas as outras e seus valores inferiores indicam que a combinação de xantana:locusta $(1: 1, \mathrm{p} / \mathrm{p})$ inferiu em prejuízo expressivo neste aspecto, situando-se na faixa entre opaco (ou não-perceptível) e moderado.

Com relação ao atributo aparência, não ocorreram diferenças significativas entre as formulações F4 e F5, mas estas diferiram das demais formulações; segundo comentários dos julgadores nas fichas de avaliação, estas duas formulações foram as que apresentaram melhor consistência e boa característica gelatinosa. Os valores médios obtidos no atributo de aparência caracterizaram estas geléias entre regular e característica à geléia; já as geléias F1, F2 e F3, ficaram na faixa entre nada característica e regular, sendo que a formulação F1, embora não diferindo significativamente, esteve bem mais distante de um resultado regular do que as outras duas formulações.

Quanto ao atributo cor, apenas as formulações F3 e F4 não diferiram significativamente da formulação controle (F5), sendo consideradas entre regular e característica; já as geléias F1 e F2 ficaram na faixa entre nada característico a geléia e regular.

A formulação F1 também diferiu significativamente das demais quanto à consistência, evidenciando o gel de consistência muito firme. No entanto, as formulações F2 e F3 apresentaram valores bem inferiores à formulação controle, o que traduz um gel fraco e de pouca consistência.
Somente a formulação F4 apresentou consistência similar à formulação controle (F5).

Pelo conjunto de resultados observa-se que a formulação F4 foi a que apresentou características mais próximas da formulação controle (F5); por outro lado, a formulação F1 foi a que obteve os resultados mais afastados da formulação controle (F5), na maioria dos atributos avaliados.

TEIXEIRA et al. [38] compararam sensorialmente geléias light de morango com geléias tradicionais, encontrando resultados muito próximos, o que permitiu indicar que a geléia light pode substituir geléias tradicionais em dietas especiais, sem perdas de seus atributos sensoriais.

\section{4 - CONCLUSÕES}

Pelos resultados deste estudo ficou evidente que a associação de diferentes gomas influiu marcadamente nas características sensoriais das geléias light de abacaxi.

A formulação F4, que consistia de xantana, carragena e locusta $(1: 1: 1, \mathrm{p} / \mathrm{p} / \mathrm{p})$, foi a geléia que apresentou os melhores resultados quando comparada às outras formulações light; entretanto, a combinação de xantana e locusta $(1: 1, \mathrm{p} / \mathrm{p})$ utilizada na formulação $\mathrm{F} 1$ apresentou um comportamento bastante diferenciado das demais combinações de gomas, com resultados sensoriais insatisfatórios.

O uso do edulcorante sucralose mostrou-se satisfatório no sentido de repor a doçura das geléias light e não inferiu em sabor característico diferenciado.

Todas as formulações de geléia light se enquadraram na legislação quanto à redução de calorias e aos limites de contaminação microbiana.

\section{5 - REFERÊNCIAS BIBLIOGRÁFICAS}

[1] ANTUNES, A. E. C.; VENDRUSCOLO, C. T.; VENDRUSCOLO, J.; MOREIRA, A . S. Produção e viscosidade de biopolímeros de cepas de xanthomonas campestris pv pruni. In: SIMPÓSIO LATINOAMERICANO DE CIÊNCIA DE ALIMENTOS, $3^{a}$ ed., 1999, Campinas. Resumo... Campinas, SP: Sociedade Brasileira de Ciência e Tecnologia de Alimentos, 1999, v. 1, p. 81.

[2] ASSOCIAÇÃO BRASILEIRA DE NORMAS TÉCNICAS - NBR 12994. Métodos de análise sensorial dos alimentos e bebidas. São Paulo, 1993, 2p.

[3] ASSOCIAÇÃO BRASILEIRA DE NORMAS TÉCNICAS - NBR 14141. Escalas utilizadas em análise sensorial de alimentos e bebidas. São Paulo, 1998, 3p.

[4] BARRETO, P. L. M.; BEIRÃO L. H. Influência do amido nas propriedades texturais de surimi de tilápia (Oreochomis, SP.). Ciência e Tecnologia de Alimentos, v. 19, n. 2, p. 183-188, 1999.

[5] BRASIL. Ministério da Saúde. Secretaria de Vigilância Sanitária. Portaria $n^{\circ} 27$ de 13 de janeiro de 1998 . Regulamento Técnico referente à Informação Nutricional Complementar. Diário Oficial da República Federativa do Brasil, Brasília, n 11-E, 16 jan. 1998.

[6] BRASIL. Ministério da Saúde. Secretaria de Vigilância Sanitária. Resolução RDC nº 12, de 02 de janeiro de 2001. 
Regulamento técnico sobre os padrões microbiológicos para alimentos. Diário Oficial da República Federativa do Brasil, Brasília, n. 7-E, 10 jan. 2001. p. 45-53.

[7] CAMPOS, A. M.; CÂNDIDO, L. M. B. Formulação e avaliação físico-química e reológica de geléias de baixo teor de sólidos solúveis com diferentes adoçantes e edulcorantes. Ciência e Tecnologia de Alimentos. Campinas, v. 15, n. 3, p. 268-278, 1995.

[8] CÂNDIDO, L. M. B.; CAMPOS, A. M. Alimentos para Fins Especiais: Dietéticos. São Paulo: Ed. Varela, 1996, $411 \mathrm{p}$.

[9] CÂNDIDO, L. M. B.; CAMPOS, A. M. Substitutos de gordura. Bol. CEPPA. Curitiba, v. 13, n. 2, p. 125-164, 1995.

[10] DZIEZAK, J.D. A focus on gums. Food Technology, Chicago, v. 43, n. 10, p. 104-116, 1989.

[11] DZIEZAK, J.D. Ingredients for sweet success. Food Technology, Chicago, v. 45, n. 3, p. 116-133, 1991.

[12] FDA (FOOD AND ADMINISTRATION). Bacteriological Analytical Manual, 6th ed. Estados Unidos, 1992.

[13] FRANCO, Guilherme. Tabela de Composição Química dos Alimentos. $8^{\mathrm{a}}$ ed. Rio de Janeiro: Livraria Atheneu, 1989, $230 \mathrm{p}$

[14] FRYER, L. C.; ARAMOUNI, F. M.; CHAMBERS IV, E. Xanthan, hydroxypropil methyl cellulose and high fructose corn syrup sensory effects in a reduced calorie syrup model. Journal of Food Science, v. 61, n. 1, p. 245-247, 252, 1996.

[15] Geléia diet - Gelifrut Ritter. On line. Disponível na Internet: <http://www.ritter.com.br/propgeli.htm> Em: 12 jun. 2000.

[16] GLIKSMAN, M. Red seaweed extracts. In: Food Hydrocolloids. Boca Raton: M. GLIKSMAN, 1983. v. 2.

[17] GRANADA, G. G.; MENDONÇA, C. B.; ZAMBIAZI, R. C. Geléias de maçã com baixas calorias. In: $9^{\circ} \mathrm{CON}$ GRESSO DE INICIAÇÃO CIENTÍFICA $/ 2^{\circ}$ ENCONTRO DA PÓS GRADUAÇÂO-UFPEL, FURG, UCPEL, Pelotas-RS, 2000. Resumos ...: Pelotas-RS, v. 2, p. 714.

[18] HAJDENWURCEL, J. R. Atlas de Microbiologia de Alimentos. São Paulo, v. 1, 1998.

[19] IBGE. Estudo Nacional da Despesa Familiar: tabela de composição dos alimentos. Rio de Janeiro: O Instituto. 1976, $172 \mathrm{p}$.

[20] INSTITUTO ADOLFO LUTZ. Normas Analíticas do Instituto Adolfo Lutz. $2^{\text {a }}$ ed. São Paulo, 1985, 371 p.

[21] INTERNATIONAL ORGANIZATION FOR STANDARDIZATION. ISO 5497: Sensory Analisys-Methodology-Guidelines for the preparation of samples for which direct sensory analisys is not feasible. Genève, 1982, 4 p.

[22] INTERNATIONAL ORGANIZATION FOR STANDARDIZATION. ISO 4121: Sensory Analisys-Methodology-Evaluation of food products by methods using scales. Genève, 1987, 8 p.

[23] INTERNATIONAL ORGANIZATION FOR STANDARDIZATION. ISO 8589: Sensory Analisys-General guidance for the design of test rooms. Genève, 1988, $9 \mathrm{p}$.

[24] INTERNATIONAL ORGANIZATION FOR STANDARDIZATION. ISO 8586-1: Sensory Analisys-General guidance for the selection, training and monitoring of assessors. Genève, 1993, 15 p.

[25] INTERNATIONAL ORGANIZATION FOR STANDARD-
IZATION. ISO 13299: Sensory Analisys-MethodologyGeneral guidance for the establishing a sensory profile. Genève, 1995, 8 p.

[26] LOBO, A. R.; SILVA, G. M. L. Aspectos Tecnológicos de Produtos de Panificação e Massas Alimentícias com Teor Calórico Reduzido. Bol. sbCTA, v. 37, n. 1, p 1-8, 2003.

[27] MENDONÇA, Carla Rosane Barboza. Sucralose e Acesulfame-k em Compotas de Pêssego com Reduzido Teor Calórico. Pelotas, 1999. Dissertação (Mestrado em Ciência e Tecnologia Agroindustrial) - Universidade Federal de Pelotas.

[28] MENDONÇA, C.; GRANADA, G.; PORTO, D.; ZAMBIAZI, R; CARVALHO, D.; CLASEN, H. Sucralose em geléias de maçã. In: CONGRESSO BRASILEIRO DE CIÊNCIA E TECNOLOGIA DE ALIMENTOS, 17, Fortaleza-CE, 2000, Resumos... Fortaleza-CE, Sociedade Brasileira de Ciência e Tecnologia de Alimentos, 2000, v. 2, p. 5.194.

[29] MENDONÇA, C. R.; RODRIGUES, R. S.; ZAMBIAZI, R. C. Açúcar mascavo em geleiadas de maçã. Ciência Rural, Santa Maria, v. 30, p. 1053-1058, 2000.

[30] NACHTIGALL, A. M., SOUZA, E. L., MALGARIM, M. B., ZAMBIAZI, R. C. Geléia light de amora-preta. Bol. CEPPA, v. 22, n. 2, p. 337-354, 2004.

[31] PASQUEL, A. Gomas: utilização e aspectos reológicos. Bol. sbCTA. Campinas, v. 33, n. 1, p. 86-97, 1999.

[32] RIBEIRO, K.O., RODRIGUES, M.I., SABADINI, E., CUNHA, R.L. Mechanical properties of acid sodium caseinate-k-carrageenan gels: effect of co-solute addition. Food Hydrocolloids, v. 18 p. 71-79, 2004.

[33] SANDERSON, G.R. Polysaccharides in foods. Food Technology, Chicago, p. 50-83, 1981.

[34] SENAI-RS. Alimentação. Fabricação de geléias e geleiadas. Porto Alegre, SENAI-RS, 1990. 61 p.

[35] STATSOFT-Statistica. Tulsa: Statsoft Inc., v. 1, 1991,935 p.

[36] STREUBEL, A., SIEPMANN, J., BODMEIER, R. Floating matrix tablets based on low density foam powder: effects of formulation and processing parameters on drug release. European Journal of Pharmaceutical Sciences, v. 18, n. 1, p. 37-4, 2003.

[37] TEIXEIRA, A. M.; SOUZA N. L.; ZAMBIAZI, R. Elaboração de geléias light. In: SIMPÓSIO DE CIÊNCIA DE ALIMENTOS, Florianópolis-SC, 2001, Resumos... Florianópolis-SC, Sociedade Brasileira de Ciência e Tecnologia de Alimentos, Regional Santa Catarina, 2001, p. QB.31.

[38] TEIXEIRA, F. S.; PAULA, P.; SILVA, P. B.; MIGUEL, V.; VERRUMA-BERNARDI, M. R. Análise sensorial de geléia light de morango em comparação com a tradicional. In: CONGRESSO BRASILEIRO DE CIÊNCIA E TECNOLOGIA DE ALIMENTOS, 17, Fortaleza-CE, 2000, Resumos... Fortaleza-CE, Sociedade Brasileira de Ciência e Tecnologia de Alimentos, 2000, v. 1, p. 3.43.

[39] TOREZAN, G. A. P.; PEZOA GARCIA, N. H. Produção de geléia de manga através de processo contínuo de fabricação, rica em sólidos da fruta e sem adição de açúcares. In: CONGRESSO BRASILEIRO DE CIÊNCIA E TECNOLOGIA DE ALIMENTOS, 17, Fortaleza-CE, 2000, Resumos... Fortaleza-CE, Sociedade Brasileira de Ciência e Tecnologia de Alimentos, 2000, v. 4, p. 11.136. 
[40] TORREZAN, R.; EIROA, M. N. U.; PFENNING, L. Identificação de microrganismos isolados em frutas, polpas e ambiente industrial. Bol. do CEPPA, v. 18, n. 1, p. 27-38, 2000.

[41] VENDRAMEL, S. M. R.; CÂNDIDO, L. M. B.; CAMPOS, A. M.. Avaliação reológica e sensorial de geléias com baixo teor de sólidos solúveis com diferentes hidrocolóides obtidas a partir de formulações em pó. Bol. CEPPA. v. 15, n. 1, p. 37-56, 1997.
[42] VANDERZANT, C.; SPLITTSTOESSER, D.F. Compendium for the Microbiological Examination of Foods. $3^{\text {rd }} \mathrm{ed}$. Washington: Public Health Association, 1992, 1219 p.

[43] Velde, F. V.; Weinbreck, F.; Edelman, M. W.; Linden, L.; Tromp, R. H. Visualisation of biopolymer mixtures using confocal scanning laser microscopy (CSLM) and covalent labelling techniques. Colloids and Surfaces B: Biointerfaces, v. 31 , n. 1-4, p 159-168, 2003. 